\title{
Prevalence of orthostatic hypotension in Parkinson's disease
}

\author{
J M Senard, S Raï, M Lapeyre-Mestre, C Brefel, O Rascol, A Rascol, J L Montastruc
}

Laboratoire de

Pharmacologie

Médicale et Clinique,

INSERM U317, Faculté

de Médecine

J M Senard

S Raï

M Lapeyre-Mestre

C Brefel

O Rascol

J L Montastruc

Service de Neurologie, Purpan University Hospital,Toulouse, France

A Rascol

Correspondence to: Professor JM Senard, Laboratoire de

Pharmacologie Médicale et Clinique, INSERM U317, Faculté de Médecine, 37

allées Jules Guesde, 31073

Toulouse cedex, France.

Received 22 September 1995 and in final revised form 12 May 1997

Accepted 22 May 1997

\begin{abstract}
Objectives-To investigate the prevalence of orthostatic hypotension and the nature of the postural events related to a fall in blood pressure in patients with Parkinson's disease.

Methods-Blood pressure was measured first in a supine position after a rest of at least $\mathbf{1 5}$ minutes and every minute during 10 minutes of an active standing up procedure. Orthostatic hypotension was considered as present when a fall of at least 20 mm $\mathrm{Hg}$ of systolic blood pressure was recorded. Postural events which occurred during the standing test were identified from a questionnaire and self reporting. Statistical analysis was performed to determine the relation between orthostatic hypotension and disease characteristics (duration, severity) and the use of antiparkinsonian drugs. Ninety one consecutive patients with Parkinson's disease (48 women, 43 men, mean age 66 (SD 9) years) participated to the study.

Results-A fall of at least $20 \mathrm{~mm} \mathrm{Hg}$ of systolic blood pressure was found in $\mathbf{5 8 . 2 \%}$ of the patients. Orthostatic hypotension was asymptomatic in $38.5 \%$ and associated with postural events in $19.8 \%$ of the patients. Symptomatic (but not asymptomatic) orthostatic hypotension was related to duration and severity of the disease and with the use of higher daily levodopa and bromocriptine doses. The analysis of the relation between the postural symptoms (and the need for standing test abortion) with the fall in systolic blood pressure allowed the identification of six clinical criteria specific of orthostatic hypotension. A direct relation between the postural changes in systolic blood pressure and the number of clinical events in this clinical scale was found. Conclusion-The frequency of orthostatic hypotension in Parkinson's disease is high and it is possible to establish a clinical rating scale which could be used to assess the effects of drugs employed in the management of orthostatic hypotension.
\end{abstract}

(F Neurol Neurosurg Psychiatry 1997;63:584-589)

Keywords: orthostatic hypotension; Parkinson's disease; postural symptoms

Autonomic features are often encountered in Parkinson's disease. They include gastrointestinal, sudoromotor, thermoregulation, and blad- der abnormalities. ${ }^{12}$ For the cardiovascular system, reduced variability of the R-R interval and impaired parasympathetic control of heart rate have been described ${ }^{3}$ as well as livedo reticularis or oedema. ${ }^{1}$ The disturbances of blood pressure regulation remain less well understood. Micieli et al reported a series of parkinsonian patients with postprandial hypotension. ${ }^{5}$ Nychthemeral variability of blood pressure was also shown to be reduced and some patients with Parkinson's disease have nocturnal supine hypertension. ${ }^{6}$ Despite evidence for the involvement of the sympathetic system in the autonomic dysfunction of Parkinson's disease, the occurrence of orthostatic hypotension in the course of the disease remains controversial. Some authors have reported the existence of orthostatic hypotension $^{4-10}$ whereas others did not find this. ${ }^{11-15}$ The reasons for these discrepancies can be summarised as follows: (1) there is no consensus for the diagnostic criteria of orthostatic hypotension, (2) medications including antiparkinsonian drugs are the most frequent cause of orthostatic hypotension, and (3) the occurrence of orthostatic hypotension often leads to the diagnosis of diseases other than idiopathic Parkinson's disease.

Thus in the present study, we prospectively investigated the prevalence of orthostatic hypotension in a series of 91 consecutive patients with Parkinson's disease. The nature of the symptoms linked to orthostatic hypotension was investigated to construct a reliable clinical scale for future clinical trials.

\section{Patients and methods}

PATIENTS

The study was performed on patients visiting the Neuropharmacological Unit of the Department of Neurology with idiopathic Parkinson's disease (according to the criteria of the United Kingdom Brain Bank ${ }^{16}$ ). Only patients with a clear and sustained response to levodopa and without recent modifications of their drug regimen (all treatments were stable for at least four weeks) were recruited in the study after they had given their informed consent. Patients who presented atypical neurological features (cerebellar or pyramidal features, prominent autonomic features, laryngeal stridor, myoclonus) suggesting that they had multiple system atrophy were excluded. ${ }^{17}$ The severity of the disease was evaluated with the Hoehn and Yahr scale. ${ }^{18}$ Associated medical conditions and daily doses of all current medications were recorded. 
MEASUREMENTS OF BLOOD PRESSURE AND HEART RATE

Blood pressure and heart rate were recorded using a semiautomatic device $\left(\right.$ Dinamap $^{\circ}$ ). Blood pressure was first measured after a 15 minute rest in a supine position. Patients were then asked to spontaneously stand up and blood pressure was recorded every minute for 10 minutes in the standing position. A decrease $\geqslant 20 \mathrm{~mm} \mathrm{Hg}$ in systolic blood pressure was chosen as the criterion for orthostatic hypotension. Blood pressure measurements were made during the morning between 0900 and 1100 for all patients. All patients took their regular morning treatment and had a regular breakfast at least two hours before measurement of blood pressure.

EVALUATION OF CLINICAL EVENTS RELATED TO STANDING POSITION

The clinical events related to orthostatism were recorded for the study day at the end of the orthostatism test. The physician asked the patient about the presence or the absence of symptoms which could be related to a fall in blood pressure during the current standing test. Recording of postural events was achieved using a questionnaire which comprised eight subjective items (lightheadedness, dizziness, postural instability, vertigo, blurred vision, fainting, asthenia, decreased hearing). For postural instability, only unusal instability was retained as this symptom could also be related to Parkinson's disease. Moreover, the need for standing procedure abortion and of return to a supine position was also rated and included in the list of events induced by postural change. The questionnaire contained repetitive items (fainting and asthenia, postural instability and dizziness) to test its internal validity and the consistency of patient responses. One unrelated item (decrease in hearing) was also included to test external validity. All additional postural symptoms not included in the questionnaire but spontaneously reported by the patients were also rated.

\section{STATISTICAL ANALYSIS}

Quantitative data are presented as means (SD) and were compared using Student's $t$ test and ANOVA. The relation between the number of clinical events and the fall in blood pressure was tested by the Pearson correlation test. Qualitative data were compared using the $\chi^{2}$ test with Yates' correction or the exact Irwin-Fisher test. Data were analysed as univariate. Only clinical events significantly related to orthostatic hypotension by $\chi^{2}$ test were used to build a global clinical assessment.
Because some of the items compared were repetitive, concordance in the response of the patients to these items was assessed by $\kappa$ coefficient, and internal consistency of the chosen items by the Cronbach $\alpha$ coefficient. A P value $<0.05$ was considered as significant.

\section{Results}

DESCRIPTION OF THE POPULATION

Ninety one consecutive patients (mean age: 66 (9) years; 48 women and 43 men) entered the study. Twenty one patients were in stage I, 49 in stage II, 19 in stage III, and two in stage IV of the Hoehn and Yahr's classification. All patients received levodopa (plus dopadecarboxylase inhibitor) at a mean daily dose of 682 (551) mg. Forty three patients were also treated with bromocriptine and five received lisuride. Some patients were taking other antiparkinsonian drugs such as anticholinergic drugs $(n=15)$ or selegiline $(n=19)$. Eleven patients had arterial hypertension and were treated with diuretic drugs $(n=6)$, angiotensin converting enzyme inhibitors $(n=3)$, or calcium antagonists $(n=4)$. No patients were taking any antihypotensive drugs and 30 received domperidone (mean daily dose: $60(20) \mathrm{mg}$ ).

\section{PREVALENCE OF ORTHOSTATIC HYPOTENSION}

A decrease of at least $20 \mathrm{~mm} \mathrm{Hg}$ in systolic blood pressure values was found in $53(58.2 \%)$ of the ninety one patients investigated. The postural fall in blood pressure was asymptomatic in $35(38.5 \%)$ and associated with at least one clinical event in 18 patients $(19.8 \%)$.

When a $20 \mathrm{~mm} \mathrm{Hg}$ decrease in systolic blood pressure was chosen as the main criteria for orthostatic hypotension, patients with orthostatic hypotension did not differ from those without it regardless of age, sex, disease duration, daily levodopa dose, or daily bromocriptine intake (table 1). The proportion of patients receiving bromocriptine was significantly higher in the group of patients with manometric orthostatic hypotension (56.6\%) than in patients without $(34.2 \%)$. There was no significant difference between patients with or without orthostatic hypotension for other antiparkinsonian drugs such as lisuride $(5.7 \%$ $v 5.3 \%)$, deprenyl $(25.7 \%$ v $18.4 \%)$, or domperidone $(18.7 \% v 14.3 \%)$. The presence of manometric orthostatic hypotension was not associated with arterial hypertension or antihypertensive drug intake (six $v$ five patients). The severity of the disease assessed using Hoehn and Yahr's classification was significantly higher in patients with than without orthostatic hypotension (table 1 ).

Table 1 Clinical characteristics of patients with Parkinson's disease without orthostatic hypotension (OH), with asymtomatic OH (defined as a systolic blood pressure (SBP) fall $\geqslant 20 \mathrm{~mm} \mathrm{Hg}$ without postural symptoms) and with symptomatic OH (both a decrease $>20 \mathrm{~mm} H \mathrm{H}$ in SBP and at least one related symptom)

\begin{tabular}{|c|c|c|c|c|c|c|c|}
\hline & Age (y) & $\%$ of men & $D D(y)$ & $H$ and $Y$ & $\begin{array}{l}\triangle S B P(m m \\
H g)\end{array}$ & $\begin{array}{l}\text { Levodopat } \\
\text { (mg/day) }\end{array}$ & $\begin{array}{l}\text { Bromocriptine } \\
\text { (mg/day) }\end{array}$ \\
\hline Without $\mathrm{OH}(\mathrm{n}=38)$ & $64(0)$ & 57.9 & $7(6)$ & I:13; II:20 III:5;IV:0 & 9 (6) & $611(521)$ & $39(17)$ \\
\hline Asymptomatic $\mathrm{OH}(\mathrm{n}=35)$ & $68(9)$ & 57.1 & 7 (6) & I:5; II:20 III:9;IV: $1^{\star}$ & $30(9)^{\star}$ & $590 \quad(412)$ & $42(19)^{\star}$ \\
\hline Symptomatic $\mathrm{OH}(\mathrm{n}=18)$ & $67(9)$ & 66.7 & $12(6)^{\star} \dagger$ & I:3;II:9 III:5;IV: $1^{\star}$ & $46(17)^{\star} \dagger$ & $1069(754)^{\star} \dagger$ & $74(26)^{\star}+$ \\
\hline
\end{tabular}

$\star \mathrm{P}<0.05 v \mathrm{OH} .+\mathrm{P}<0.05 v$ patients with asymptomatic $\mathrm{OH}$.

Values are mean $(\mathrm{SD}) . \mathrm{DD}=$ disease duration; $\mathrm{H}$ and $\mathrm{Y}=\mathrm{Hoehn}$ and $\mathrm{Yahr}$ stage; $\Delta \mathrm{SBP}=$ systolic blood pressure fall when standing; Levodopa $=$ daily dose + dopa-decarboxylase inhibitor. 
Table 2 Number of patients with and without orthostatic hypotension (OH) (defined as a fall of at least $20 \mathrm{~mm} \mathrm{Hg}$ systolic blood pressure) presenting at least one symptom related to orthostatism or needing standing test abortion

\begin{tabular}{llll}
\hline & $\begin{array}{l}\text { With OH } \\
(n=53)\end{array}$ & $\begin{array}{l}\text { Without OH } \\
(n=38)\end{array}$ & RR (95\% CI) \\
\hline Decreased hearing & 1 & 1 & $0.86(0.21-3.46)$ \\
Lumbalgia & 1 & 0 & $\mathrm{NC}$ \\
Thirst & 1 & 0 & $\mathrm{NC}$ \\
Dazzling & 2 & 0 & $\mathrm{NC}$ \\
Tired legs & 1 & 0 & $\mathrm{NC}$ \\
Heavyheadedness & 2 & 0 & $\mathrm{NC}$ \\
Standing test abortion & 4 & 0 & $\mathrm{NC}$ \\
Lightheadedness & $6^{\star}$ & 0 & $\mathrm{NC}$ \\
Blurred vision & $7^{\star}$ & 0 & $\mathrm{NC}$ \\
Asthenia or fainting & $10^{\star}$ & 0 & $1.95(1.50-2.53)$ \\
Dizziness or vertigo & $18^{\star}$ & 1 & $1.95(1.50-2.53)$ \\
Dysequilibrium or postural instability & $18^{\star}$ & 1 & \\
\hline
\end{tabular}

^Found to be related to blood pressure fall. $\mathrm{NC}=$ not calculable.

The repetitive symptoms were regrouped according to the Cronbach $\alpha$ coefficient (see methods).

Similar results were obtained for patients with non-symptomatic orthostatic hypotension (systolic blood pressure decrease $\geqslant 20 \mathrm{~mm} \mathrm{Hg}$ without postural symptoms) when compared with those without orthostatic hypotension (table 1 ). In this group, $71.4 \%$ of the patients received bromocriptine, $5.7 \%$ lisuride, and $25.7 \%$ deprenyl. The number of patients receiving antihypertensive drugs or of patients being treated with domperidone was not different from that in the group who did not present a fall in systolic blood pressure during the standing test.

Patients with symptomatic orthostatic hypotension (defined as both a decrease of at least $20 \mathrm{~mm} \mathrm{Hg}$ in systolic blood pressure and the presence of at least one postural symptom) had a significantly longer duration of disease and received larger daily levodopa and bromocriptine doses than patients without orthostatic hypotension or with non-symptomatic orthostatic hypotension. The severity of the disease was higher than in patients without orthostatic hypotension but similar to that of patients with non-symptomatic orthostatic hypotension (table 1). The daily doses of levodopa and of bromocriptine were significantly higher in patients with symptomatic orthostatic hypotension than in patients with asymptomatic or without orthostatic hypotension. The proportion of patients with symptomatic orthostatic hypotension receiving bromocriptine $(27.8 \%)$ was similar to that in patients without orthostatic hypotension $(34.2 \%)$ but significantly lower than the proportion of patients with asymptomatic orthostatic hypotension and bromocriptine treatment $(71.4 \%)$. For the other indices studied (age, sex, and other antiparkinsonian medications), no significant difference was noticed. In patients with symptomatic orthostatic hypotension, the fall in systolic blood pressure during orthostatism (46 (17) $\mathrm{mm} \mathrm{Hg}$ ) was significantly higher than in patients without postural symptoms (9 (6) mm $\mathrm{Hg})$ or with non-symptomatic orthostatic hypotension (30 (9) $\mathrm{mm} \mathrm{Hg}$ ).

CLINICAL EVENTS RELATED TO ORTHOSTATIC HYPOTENSION

In the group of patients without manometric orthostatic hypotension $(\mathrm{n}=38)$, only four reported one symptom during the standing procedure. Fifteen different clinical events occurred during the standing procedure in the 18 patients with symptomatic orthostatic hypotension. Among these, only six symptoms were significantly related to the manometric criteria chosen for orthostatic hypotension (table 2). By contrast, fainting and asthenia as well as the need for standing test abortion were not related to the presence of manometric orthostatic hypotension. This was probably due to the limited number of symptomatic patients included in this series. The frequencies for repetitive symptoms (dysequilibrium and postural instability, asthenia and fainting) included in the questionnaire to test its internal validity were not significantly different. This indicates that patients were consistent in answering the questionnaire as patients reporting asthenia or dysequilibrium also reported unusual fainting and postural instability respectively $(93.5 \%$ (95\% confidence interval (95\% CI) $81.1-98.3$ ) and $91.3 \%$ of concordance (95\% CI 78.3-97.2) respectively). Furthermore, as hypothetised, only one patient with and one without orthostatic hypotension complained of a decrease in hearing suggesting that the questionnaire was able to discriminate events related or not related to orthostatic hypotension.

Finally, taking account of the consistency of response, repetitive items were regrouped and a global assessment including six postural events (blurred vision, postural instability or dysequilibrium, fainting or asthenia, need for standing test abortion, dizziness or vertigo and lightheadedness) was established. The frequency of the need for abortion of the standing test did not reach significance but it was included in the clinical assessment because it represents an index of severity of orthostatic hypotension. The internal consistency of these six criteria was shown by the Cronbach $\alpha$ coefficient of 0.856 . When investigating the diagnostic value of these criteria for the diagnosis of orthostatic hypotension, we found that when at least one postural symptom was present, specificity was high $(94.7 \%)$ but sensitivity was low $(34 \%)$. Specificity was increased $(100 \%)$ when at least two clinical events were present. Moreover, postural symptoms correlated positively with the quantitative importance of the fall in systolic blood pressure $(r=0.541)$. There was no relation between the clinical score and Parkinson's disease characteristics or current treatments (data not shown).

\section{Discussion}

The present data suggest that orthostatic hypotension is often present in Parkinson's disease. Symptomatic orthostatic hypotension is related to duration of disease, daily levodopa and bromocriptine dose, and the importance of systolic blood pressure fall during the standing procedure. Moreover, it was possible to identify postural events directly related to the fall in systolic blood pressure and to define a clinical score reflecting the severity of orthostatic hypotension.

Sir James Parkinson in his first description of shaking palsy reported the association of motor 
features with symptoms suggesting the involvement of the autonomic nervous system..$^{19}$ More recently several investigators have reported the existence of autonomic disorders in the course of Parkinson's disease (for reviews see Korc$\mathrm{zyn}^{1}$ and Streifler $e t a l^{2}$ ). Lewy bodies have been found in brain regions involved in activity of the autonomic nervous system, such as the locus coeruleus, dorsal vagal nucleus, and intermediolateral nucleus but also in the sympathetic ganglia and in the myenteric and submucosal plexus of the digestive tract. Despite the clinical and pathological evidence indicating that autonomic dysfunction is part of Parkinson's disease, many controversies remain concerning the existence of orthostatic hypotension as a feature of autonomic dysfunction in the disease. In 1965, Fichefet et al reported a case of idiopathic Parkinson's disease associated with severe orthostatic hypotension. ${ }^{7}$ More recently, other authors have reported similar clinical findings. ${ }^{4-10}$ However, some investigators deny the association of Parkinson's disease with orthostatic hypotension. ${ }^{11-15}$ The reasons for these discrepancies are multiple and will be discussed separately.

The most important one is that no definite consensus exists for the diagnosis criteria for orthostatic hypotension. A systolic blood pressure fall $\geqslant 20 \mathrm{~mm} \mathrm{Hg}$ is the most often used criterion as it was shown to be associated with an increased risk of falls, syncope, or mortality in hypertensive people. For other authors, the added presence of postural symptoms is required. The effect of the different diagnosis criteria on the prevalence of orthostatic hypotension in large groups of people over 65 years of age was recently illustrated. In three epidemiological studies, the prevalence of a decrease in systolic blood pressure $\geqslant 20 \mathrm{~mm}$ $\mathrm{Hg}$ was found to be between $7.9 \%$ and $28 \%$ but only between $2 \%-3 \%$ when the fall in systolic blood pressure was associated with postural symptomatology. ${ }^{20-22}$ For Parkinson's disease, as far as we know, only a few studies have reported the prevalence of orthostatic hypotension. Singer et al found a prevalence of orthostatic dizziness in $22 \%$ of 48 parkinsonian patients but they did not measure changes in blood pressure induced by postural change. ${ }^{23}$ Uono found orthostatic hypotension in $43 \%$ of a series of 80 patients. ${ }^{8}$ Recently, Netten et al encountered a fall $>20 \mathrm{~mm} \mathrm{Hg}$ of systolic blood pressure in four out of five patients with Parkinson's disease diagnosed as having autonomic failure. ${ }^{24}$

In the present study, we investigated the prevalence of orthostatic hypotension using both manometric and clinical criteria. When using just a decrease of at least $20 \mathrm{~mm} \mathrm{Hg}$ in systolic blood pressure, we found that $58.2 \%$ of the patients from our study had orthostatic hypotension. This percentage was significantly lower when orthostatic hypotension was defined by both a decrease in systolic blood pressure $\geqslant 20 \mathrm{~mm} \mathrm{Hg}$ and the presence of at least one postural symptom (19.8\%). Although our study was uncontrolled, the prevalence seems to be much higher than the prevalence reported in the general population older than 65 years of age. $^{20-22}$ This high prevalence of orthostatic hypotension cannot be ascribed to age of the patients as it was similar to that of large epidemiological studies. The relation between age and prevalence of orthostatic hypotension remains a matter of debate. Two recent works failed to find any influence of age on changes in systolic blood pressure during orthostatism. ${ }^{325}$ Our results cannot be compared with those of Gross et al ${ }^{9}$ or Kuno et $a l^{14}$ as these authors used a passive head up tilt test to investigate blood pressure changes whereas we used active standing. They are more consistent with the $23 \%$ prevalence of orthostatic hypotension in a group of 23 patients with Parkinson's disease reported by Netten et al. ${ }^{24}$ Other factors are now recognised as lowering blood pressure, such as food ingestion, exercise, and heat stress. ${ }^{26} \mathrm{~A}$ role for these different factors in our study can be discarded as all blood pressure measurements were performed at least two hours after a normal breakfast. Moreover, patients were rated in a room with controlled temperature and were tested after a period of rest of at least 15 minutes in a supine position.

The second reason explaining the discrepancies on the existence or not of orthostatic hypotension in Parkinson's disease is that most orthostatic hypotension is due to the adverse effects of drugs. Despite the fact that no data are available on the prevalence of drug induced orthostatic hypotension, there have been many reports on the role of antihypertensive medications and of psychotropic drugs including antiparkinsonian medications. However, antihypertensive medications were not associated with orthostatic hypotension in the study of Räihä et $a .^{22}$ The use of antihypertensive drugs does not explain the high prevalence of orthostatic hypotension in our study as a similar number of patients with and without orthostatic hypotension were taking them. We found that patients with Parkinson's disease with symptomatic orthostatic hypotension were taking larger daily levodopa doses than patients with non-symptomatic orthostatic hypotension or without orthostatic hypotension. The effects of levodopa (plus dopa-decarboxylase inhibitors) on cardiovascular adaptation during standing in patients with Parkinson's disease have been repeatedly investigated but remain a matter of discussion. Some studies failed to find any effect of acute levodopa administration on cardiovascular responses to standing. ${ }^{12}{ }^{27-29}$ On the other hand, Camerlingo et al reported that a two year continuous treatment with levodopa was associated with a decrease in blood pressure adaptation during tilting. ${ }^{30}$ Although some authors stated that orthostatic hypotension is related to more advanced stages of the disease, they indicated that chronic antiparkinsonian medication did not account for changes in blood pressure during standing. ${ }^{41}$ Thus despite some controversy it is possible that high doses of levodopa may increase the risk of symptomatic orthostatic hypotension. ${ }^{32}$ However, the larger daily dose of levodopa in patients with orthostatic hypotension can also be explained by other factors 
related to the disease. Orthostatic hypotension was associated with a longer duration of the disease which is itself related to higher severity of Parkinson's disease and to higher levodopa dosages. Taken together, these data indicate that there is no clear evidence for a connection between high dosage of levodopa on the incidence of orthostatic hypotension. For bromocriptine, the results are similar to those of levodopa. More patients with orthostatic hypotension were receiving bromocriptine and the presence of postural symptoms was associated with high daily doses. This finding is probably relevant for the high prevalence of orthostatic hypotension as bromocriptine has repeatedly been reported to impair cardiovascular adaptation during standing ${ }^{33}$ in accordance with its sympatholytic effects. ${ }^{34}$ However, this effect is usually found after acute or subchronic administration and not after chronic exposure to the drug. ${ }^{28}$ Furthermore, the percentage of patients receiving bromocriptine was similar in the groups with symptomatic orthostatic hypotension or without orthostatic hypotension. A difference in the number of patients treated with domperidone or in the dose of this peripheral dopaminergic antagonist used does not explain the results found as these two indices were similar in the different groups. Whatever the exact responsibility of antiparkinsonian drugs, the selection for this study of patients with a stable dose regimen for at least four weeks probably eliminated most of the drug induced orthostatic hypotension which is usually seen during the first days after drug introduction or increase in dosage. Furthermore, in none of these patients had antiparkinsonian drug dosage been lowered in the past because of orthostatic hypotension.

Another crucial problem with autonomic failure in Parkinson's disease is that the occurrence of orthostatic hypotension in the course of the disease makes the diagnosis of idiopathic Parkinson's disease uncertain and evokes other degenerative diseases such as multiple system atrophy. As repeatedly shown by postmortem examinations, there is no clear clinical criteria allowing physicians to discard multiple system atrophy from idiopathic Parkinson's disease, especially in the early stages of the disease. ${ }^{17}{ }^{35}$ To try to avoid this problem, we only included patients with extrapyramidal features who did not present additional neurological features and who had a sustained good response to levodopa. We found that orthostatic hypotension was more frequent in patients with longer duration of disease. Although we did not exhaustively investigate autonomic function in these patients, our results agree with the data of Orskov et $a l^{4}$ and Gert van Dijk et $a l^{\beta 1}$ and can be interpreted as the progressive extension of Lewy bodies to autonomic structures.

The second part of this study aimed to identify symptoms specifically related to the fall in systolic blood pressure to define a clinical score usuable in clinical trials. The clinical symptoms related to orthostatic hypotension are claimed to be non-specific and to reflect a decrease in cerebral blood flow during standing. However, various investigators have shown that the most frequent symptoms of orthostatic hypotension, such as dizziness, can also be related to other medical conditions such as cataracts or transient ischaemic attacks. ${ }^{20}{ }^{21}$ On the other hand, Ensrud et al showed that systolic hypertension, which is a known risk factor for orthostatic hypotension, is not associated with an increased prevalence of dizziness. ${ }^{21}$ Furthermore, analysis of symptoms related to orthostatic hypotension is complicated when patients have chronic neurological diseases responsible for alteration of walking or of postural balance such as parkinsonism. In the present study, we tried to identify the symptoms occurring during standing procedures which were related to a fall in systolic blood pressure. Only six symptoms were found to be related both to the postural procedure and a decrease in systolic blood pressure $\geqslant 20 \mathrm{~mm} \mathrm{Hg}$ (lightheadedness, blurred vision, dizziness, vertigo, dysequilibrium, and unusual postural instability). Because of the limited number of symptomatic patients, the frequencies for other symptoms such as asthenia, faintings, and need for standing test abortion did not reach significance. This could also be due to the presence in the questionnaire of repetitive items included to test its internal consistency. When repetitive symptoms were regrouped together, we found that the presence of dysequilibrium or unusual postural instability, dizziness or vertigo, blurred vision, lightheadedness, and asthenia or fainting were significantly related to a fall in systolic blood pressure $\geqslant 20 \mathrm{~mm} \mathrm{Hg}$. The need for standing test abortion was also retained to build the clinical scoring system as it can be considered as an index for severity of orthostatic hypotension. Once again, its low frequency is clearly related to the reduced number of symptomatic patients we identified in this series. The presence of at least one symptom was highly specific but not very sensitive for the presence of orthostatic hypotension whereas the presence of two symptoms gave higher specificity. The number of clinical symptoms was found to be related to the extent of the fall in systolic blood pressure during the standing procedure and thus probably give a good idea of the severity of orthostatic hypotension. This particular finding in patients with Parkinson's disease differs from what is reported in less well defined surveys, in which no relation could be established between the fall in blood pressure and the occurrence of symptoms. ${ }^{20}$ These results allow us to make some comments on the correct criteria for orthostatic hypotension. When considering only a manometric decrease in systolic blood pressure, patients reaching a decrease $\leqslant 30 \mathrm{~mm}$ $\mathrm{Hg}$ never exhibited postural symptoms. As the variability of the fall in systolic blood pressure during the standing test is high, choosing, as sole criterion for orthostatic hypotension, a decrease $\geqslant 20 \mathrm{~mm} \mathrm{Hg}$ in systolic blood pressure is probably wrong as it would select many patients without established orthostatic hypotension. To increase the quality of the diagnosis, we suggest that a more adequate way to define orthostatic hypotension would be a 
decrease of $\geqslant 30 \mathrm{~mm} \mathrm{Hg}$ in systolic blood pressure as we did in previous studies ${ }^{6}$ or a decrease of $\geqslant 20 \mathrm{~mm} \mathrm{Hg}$ associated with one of the related symptoms.

In conclusion, we report a high prevalence of orthostatic hypotension in Parkinson's disease. Orthostatic hypotension in Parkinson's disease is associated with a long evolution of the disease and with a high levodopa and bromocriptine regimen but not with age, other medications, or other medical conditions. The careful investigation of the postural symptoms related to the fall in blood pressure suggests that a clinical score of severity can be constructed and used-for instance, to check the efficacy of putative antihypotensive drugs. However, this will need further studies on larger surveys to test its validity and reproductibility.

1 Korczyn AD. Autonomic nervous system disturbances in Parkinson's disease. In: Streifler MB, Korczyn AD, Melamed E, Youdim MBH, eds . Advances in neurology: Parkinson's disease: anatomy, pathology and therapy. New Parkinson's disease: anatomy, pathol

2 Takahashi A. Autonomic nervous system disorders in Parkinson's disease. Eur Neurol 1991;31(suppl 1):41-7.

3 Kuroiwa Y, Shimada Y, Toyokura Y. Postural hypotension and low R-R interval variability in parkinsonism, spinocerebellar degeneration, and Shy-Drager syndrome. Neurology 1983;33:463-7.

4 Orskov L, Jakobsen J, Dupont E, de Fine Olivarius B, Christensen NJ. Autonomic function in parkinsonian patients relates to duration of disease. Neurology 1987;37: 1173-8.

5 Micieli G, Martignoni E, Cavallini A, Sandrini G, Nappi G. Postprandial and orthostatic hypotension in Parkinson's disease. Neurology 1987;37:383-93.

6 Senard JM, Chamontin B, Rascol A, Montastruc JL. Ambulatory blood pressure in patients with Parkinson's disease without and with orthostatic hypotension. Clin Auton Res 1992;2:99-104.

7 Fichefet JP, Sternon JE, Franken L, Demanet JC, Vanderhaegen JJ. Etude anatomo-clinique d'un cas d'hypotension haegen JJ. Etude anatomo-clinique d'un cas d'hypotension
orthostatique idiopathique. Acta Cardiol 1965;20:332-48.

8 Uono Y. Parkinsonism and autonomic dysfunction. Auton Nerv Syst 1993;10:163-70.

9 Gross M, Bannister R, Godwin-Austen R. Orthostatic hypotension in Parkinson's disease. Lancet 1972;i:4-18.

10 Senard JM, Valet P, Durrieu G, Berlan M, Tran MA, Montastruc JL, et al. Adrenergic supersensitivity in parkinsonians with orthostatic hypotension. Eur $\mathcal{F}$ Clin Pharmacol 1990;20:613-9.

11 Aminoff MJ, Wilcox CS, Slatter JD. Autonomic deficits in paralysis agitans and in the Shy-Drager syndrome. Acta Neurol Scand 1972;51(suppl): 105-7.

12 Sachs C, Berglund B, Kaijser L. Autonomic cardiovascular responses in parkinsonism: effect of levodopa with dopa-decarboxylase inhibition. Acta Neurol Scand 1985;71: $37-42$

13 Turrka JT, Tolonen U, Myllylä VV. Cardiovascular reflexes in Parkinson's disease. Eur Neurol 1987;26:104-12.
14 Kuno S. Parkinson's disease and its autonomic disorders. Modern Physician 1989;9:1214-9.

15 Sandroni P, Ahlskog JE, Fealey RD, Low PA. Autonomic involvement in extrapyramidal and cerebellar disorders. Clin Auton Res 1991;1:147-55.

16 Gibb WRG, Lees AJ. The relevance of the Lewy body to the pathogenesis of idiopathic Parkinson's disease. F Neurol Neurosurg Psychiatry 1988;51:745-52.

17 Wenning GK, Shlomo YB, Magalhaes M, Daniel SC, Quinn NP. Clinical features and natural history of multiple system atrophy. Brain 1994;117:835-45.

18 Hoehn NH, Yahr MD. Parkinsonism: onset, progression, and mortality. Neurology 1967;17:427-42.

19 Parkinson J. An essay on the shaking palsy. London: Sherwood, Neely, and Jones, 1817.

20 Rutan GH, Hermanson B, Bild DE, Kittner SJ, Laban F, Tell GS. Orthostatic hypotension in the older adults. The cardiovascular health study. Hypertension 1992;19:508-19.

21 Ensrud KE, Nevitt MC, Yunis C, Hulley SB, Grimm RH, Cummings SR. Postural hypotension and postural dizziness in elderly women. The study of osteoporotic fractures. Arch Intern Med 1992;152:1058-64.

22 Räihä I, Luutonen S, Piha J, Seppänen A, Toikka T, Sourander L. Prevalence, predisposing factors, and prognostic importance of postural hypotension. Arch Intern Med 1995;155:930-5

23 Singer C, Weiner WJ, Sanchez-Ramos JL. Autonomic dysfunction in men with Parkinson's disease. Eur Neurol 1992;32:134-40.

24 Netten PM, De Vos K, Horstink MWIM, Hoefnagels WHL. Autonomic dysfunction in Parkinson's disease, tested with a computerized method using Finapres device. Clin Auton Res 1995;5:85-9.

25 Basset AM, Tomaselli CM, Hoffler WG. Cardiovascular responses to postural changes: differences with age for men and women. F Clin Pharmacol 1994;34:394-402.

26 Mathias CJ. Orthostatic hypotension: causes, mechanisms, and influencing factors. Neurology 1995;45(suppl 5):S611.

27 Senard JM, Verwaerde, Rascol O, Montastruc JL. Effects of acute levodopa administration on blood pressure and heart ate variability in never treated parkinsonians. Hypertens Res 1995;18(suppl 1):S1-3.

28 Durrieu G, Senard JM, Tran MA, Rascol A, Montastruc JL. Effects of levodopa and bromocriptine on blood pressure Effects of levodopa and bromocriptine on blood pressure harmacol 1991;14:84-90.

29 Meco G, Pratesi L, Bonifati V. Cardiovascular reflexes and autonomic dysfunction in Parkinson's disease. 7 Neurol 1991;238:195-9.

30 Camerlingo M, Ferraro B, Gazzaniga GC, Casto L, Cesana BM, Mamoli A. Cardiovascular reflexes in Parkinson's disease: long term effects of levodopa treatment on de novo patients. Acta Neurol Scand 1990;81:346-8.

31 Gert van Dijk J, Haan J, Zwinderman K, Kremer B, van Hilten BJ, Roos RAC. Autonomic nervous system dysfunction in Parkinson's disease: relationships with age, medication, duration, and severity. 7 Neurol Neurosurg Psymedication, duration, and

32 Goetz CG, Lutge W, Tanner CM. Autonomic dysfunction in Parkinson's disease. Neurology 1986;36:73-5.

33 Mets TF. Drug-induced orthostatic hypotension in older patients. Drugs Aging 1995;6:219-228.

34 Lockhandwala MF, Barret RJ. Cardiovascular dopamine receptor: physiological pharmacological and therapeutic implications. F Auton Pharmacol 1982;3:189-215.

35 Hughes AJ, Daniel SE, Kilford L, Lees AJ. Accuracy of clinical diagnosis of idiopathic Parkinson's disease: a clinico-pathological study of 100 cases. $\mathcal{F}$ Neurol Neurosurg Psychiatry 1992;55:181-4. 